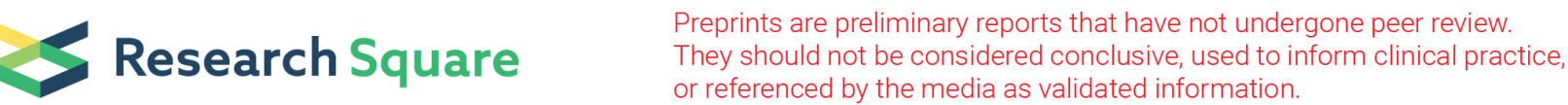

\section{Eccentricity of Aortic Annulus and Distance of Origin of Left Main and Right Coronary Arteries from Adjacent Aortic Leaflets in Non-Stenotic Aortic Valves by TEE 3D Echocardiography}

Hakimeh Sadeghian ( $\square$ sadeghianhakimeh@yahoo.com )

Arash Jalal omidi

Tehran University of Medical Sciences

Arash Jalali

Tehran Heart Center

mohammad moein ashrafi

Islamic Azad University of yazd https://orcid.org/0000-0001-5831-7447

\section{Research}

Keywords: Transesophageal 3D Echocardiography (TEE), Aortic Stenosis (AS), Aortic Annulus (AA)

Posted Date: February 19th, 2020

DOI: https://doi.org/10.21203/rs.2.24003/v1

License: (c) (i) This work is licensed under a Creative Commons Attribution 4.0 International License.

Read Full License

Version of Record: A version of this preprint was published at Cardiology and Cardiovascular Medicine on January 1st, 2022. See the published version at https://doi.org/10.26502/fccm. 92920255. 


\section{Abstract}

Background: It has been identified that aortic annulus (AA) is not completely round in normal and stenotic aortic valves and some studies invesigated the eccentricity of AA by some modalities.

Aims: To compare size of aortic valve annulus in different views via transesophageal (TEE) 3D echocardiography in patients without aortic stenosis (AS).

Method: 31 patients underwent TEE3D without AS. Full-volume of aortic valve was saved by TEE in short and long-axis views in single beat. AA was measured in 3 planes: two orthogonal lines in short-axis plane(D1 \& D2), sagittal(D3) and coronal(D4)planes, diameter of left ventricular outflow tract 5-10 mm below AA was measured in sagittal and coronal views (D5 and D6). Area and perimeter of AA were measured in short-axis plane in hing points of aortic cusps and the annulus according to area and perimeter was calculated. These different AA sizes were compared. Distance of left main (LM) and right coronary arteries (RCA) from adjacent aortic leaflets and length of left coronary cusp were measured. All measurements were done in mid-systole.

Results: From, 31 patients(51.5\% F), age $50.61 \pm 10.78$, median Left Ventricular Ejection Fraction (LVEF) was $55 \%$. AA in sagittal is smaller than coronal view, mean difference $=1.97 \mathrm{~mm}, \mathrm{P}=0.002$.D1 is smaller than D3, D2 is not different with D4. D perimeter is larger than $D$ area and $D$ sagittal, mean difference $=0.7$ and $0.9 \mathrm{~mm}$ respectively, $\mathrm{P}<0.05$ and smaller than $\mathrm{D}$ coronal, mean difference is $1 \mathrm{~mm}$. The difference between D area and D3 is not significant. D3 and D4 are not significantly different with D5 and D6. D perimeter is not different with mean D1\&D2 and D3\&D4.Mean distance of LM ostium and RCA from adjacent aortic leaflets is $14.2 \pm 2.97$ and $15.25 \pm 3.60 \mathrm{~mm}$ respectively. Mean length of left coronary cusp is $12.75 \pm 2.1 \mathrm{~mm}$.

Conclusion: AA is not completely round in non stenotic aortic valves and is larger in coronal than sagittal view, D perimeter is not different with mean annulus in sagittal and coronal views.

\section{Background:}

It has been identified that aortic annulus (AA) is not completely round in normal and stenotic aortic valves, some studies have described the eccentricity of AA both by CT and by 3D echocardiography[1]. Traditionally, AA is measured in sagittal view before transaortic valve implantation (TAVI) and final device size should be oversized compared to annular diameter. Most procedures are performed according to the annular diameter measurement by 2D Transthoracic Echocardiography (TTE) or transoesophageal echocardiography (TOE), but these methods assume annular circularity, which may result in erroneous dimensions in patients whose annuli are more ovals shaped. This limitation can be overcome using multiplanar tools of either 3D TEE or multi-slice computed tomography (MSCT) [2].

But the precise detection of AA in different echocardiographic views, diameter according to perimeter and area, distance of left main (LM) and right coronary artery (RCA) ostia from adjacent AA and length of left 
coronary cusp by 3D echo main to be determined[3].

The aim of this study is to measure AA in different views, and to measure distance of LM and RCA ostia from AA and length of left coronary cusp and diameter of left ventricular outflow tract (LVOT) in sagittal and coronal views in non-stenotic aortic valves.

\section{Methods:}

31 patients underwent TEE 3D without significant aortic stenosis (AS), mean aortic valve gradient was < $10 \mathrm{mmHg}$. Full-volume of aortic valve was saved by TEE 3D in short-axis and long-axis views.

AA was measured in four diameters. For measurement of AA, TEE 3D short-axis view by full volume was used in single beat, but if view was poor and not adequate for proper measurement, TEE long-axis 3D fullvolume was used.

If TEE 3D short-axis full volume view was used, red, blue and green (RBG) upright modality was selected and then mid systole was chosen, in sagittal view (right upper view, red plane),blue line was put align with aorta and green line is put just at hinge point of aortic cusps. Then in left lower view (blue plane), red line was put align with aorta and green line was put in hing point of aortic cusps, D1 and D2 were measured in left upper view (green plane, short-axis view), D3 and D5 were measured in right upper view (red plane, sagittal view) and D4 and D6 were measured in left lower view (blue plane, coronal view).Perimeter and area were measured in short-axis view [1].

If TEE long-axis full volume 3D view was used, RBG upright modality was used and then by grabbing the blue line in sagittal view and rotating 90 degree counter clock wise, the right upper view is coronal view and left lower view is short-axis view and the measurement were done like described above[1].

The following measurements were done in above described views (Fig. 1):

D1: AA in TEE short-axis view between junction of left and non-coronary cusps and hinge point of right coronary cusp.

D2: AA in TEE short-axis view between hinge point of left and commissure of right and non-coronary cups.

D3 : AA in TEE sagittal view.

D4: AA in TEE 3D coronal view.

D5: LVOT diameter 5-10 mm below AA in TEE sagittal view (below D3).

D6: LVOT diameter 5-10 mm below AA in coronal view (below D4).

Perimeter: Circumference of AA in short-axis view (cm). 
Area: Area of AA in short-axis view $\left(\mathrm{cm}^{2}\right)$.

Diameter of AA according to perimeter: Perimeter of $A A / \pi$.

Diameter of AA according to area: 2 *AA area $/ \pi$.

LM distance from AA: The distance between $L M$ and AA in coronal view.

RCA distance from AA: The distance between RCA and AA in TEE sagittal view.

Length of left coronary cusp: The length of left coronary cusp in TEE coronal view.

All measurements are performed in mid- systole.

\section{Statistical Analysis}

The Statistical Software Package (SPSS for Windows, version 22, SPSS Inc, Chicago, Illinois, USA) was used for the statistical analyses. The continuous variables are presented as mean $\pm S D$, and the categorical variables are expressed as percentages. The continuous variables were compared using the Student t-test or the Mann-Whitney test, if needed. The categorical variables were compared using the $\chi 2$ or the Fisher exact test. a $\mathrm{P}$ value of equal to or smaller than 0.05 was considered statistically significant. To assess reproducibility of data, data of 5 patients were randomly measured by the second observer for inter-observer variability control and by the first observer at an interval of two weeks for intra-observer variability. Inter- and intra-observer variability was calculated via dividing the mean difference between observations by their average measurement[4].

\section{Inter and Intra observer variability:}

Inter and intra observer variability was as follow:

D1: 0.37 and 0.46 ,

D2: 0.12 1nd 0.11,

D3: 0.47 and 0.21 ,

D4 was 0.04 and 0.08 ,

Distance of RCA: 0.06 and 0.06 ,

Distance of LM: 0.08 and 0.08 ,

Length of LM: 0.06 and 0.09,

D perimeter: 0.14 and 0.12 ,

D area:0.19 and 0.21 respectively. 


\section{Results:}

From, 31 patients (48.5\% male), mean age $50.61 \pm 10.78$, median LVEF was 55\%, (45\%, 25 percentile and $55 \%, 75$ percentile), mean aortic gradient was $5.43 \pm 2.06 \mathrm{mmHg}$. Median PAPs $=33.5 \mathrm{mmHg}(30 \mathrm{mmHg}$ 25 percentile and $43 \mathrm{mmHg} 75$ percentile). One patient had severe Al and 3 had severe MR (table 1).

$\mathrm{D} 1$ is smaller than $\mathrm{D} 3,22.44 \pm 2.62$ vs $23.06 \pm 2.91 \mathrm{~mm}$, mean difference is $0.61 \mathrm{~mm}, \mathrm{P}=0.02$.

The difference between D2 and D4 is not statistically significant, $24.92 \pm 3.15$ vs $25.03 \pm 3.69 \mathrm{~mm}, \mathrm{P}=$ 0.828 .

D3 is smaller than D4, $23.06 \pm 2.91 v s 25.03 \pm 3.69 \mathrm{~mm}$, mean difference $=1.97 \mathrm{~mm}, \mathrm{P}=0.002$.

Empiric distribution of difference between D4 and D3 in each subject is demonstrated by Fig. 2, median difference is $1.9 \mathrm{~mm}$, maximum difference is $9.3 \mathrm{~mm}$ and minimum difference is $-3.5 \mathrm{~mm}$ (It means that D3 is larger than D4).In $71.9 \%$ of subjects, D4 is larger than D3 and $28.1 \%$ D4 is smaller than D3.

The difference between D3 and D5 is not statistically significant, $23.06 \pm 2.91 \mathrm{vs} 22.39 \pm 3.12 \mathrm{~mm}$, mean difference is $0.66 \mathrm{~mm}, \mathrm{P}=0.07$.

The difference between D4 and D6 is not statistically significant, $25.03 \pm 3.69 \mathrm{vs} 24.25 \pm 5.70$, mean difference is $0.88 \mathrm{~mm}, \mathrm{P}=0.70$

The difference between $D$ area and $D 3$ is not statistically significant,23.39 \pm 2.58 vs $23.06 \pm 2.87, P=0.61$. $D$ area is smaller than $D$ coronal,mean difference is $1.7 \mathrm{~mm}, \mathrm{P}=0.001$

D perimeter is larger than $D$ area, $24.00 \pm 2.69$ vs $23.39 \pm 2.58$ mean difference $=0.70 \mathrm{~mm}, P=0.005$.

D perimeter is smaller than D4 (D coronal), $24.00 \pm 2.69 \mathrm{vs} 25.03 \pm 3.69 \mathrm{~mm}$,mean difference is $1.03 \mathrm{~mm}$, $P=0.037$.

D perimeter is larger than D3 (sagittal), $24.00 \pm 2.69$ vs $23.06 \pm 2.91$, mean difference is $0.93 \mathrm{~mm}, \mathrm{P}=$ 0.019 .

Mean D1 and D2 is not different with mean D3 and D4,P = 0.39.

Annulus according to area is not different with mean $D 1 \& D 2, P=0.18$,but is significantly smaller than mean $D 3 \& D 4, P=0.049$.

Annulus according to perimeter is not different with mean D1\&D2 and mean D3\&D4,P $=0.35$ and $P=0.88$ respectively.

Mean distance between LM ostium and AA is $14.2 \pm 2.94 \mathrm{~mm}$.

Mean distance between right coronary ostium and $A A=15.25 \pm 3.60 \mathrm{~mm}$. 
Mean length of left coronary cusp $=12.75 \pm 2.12 \mathrm{~mm}$.

Distance of LM from AA is longer than distance between RCA and AA, mean difference $=1.05, P=0.052$.

Length of left coronary cusp is shorter than distance of $L M$ from AA, mean difference $=1.05, P=0.001$ (table 2).

\section{Discussion:}

The results of our study showed that the coronal diameter of AA is about $2 \mathrm{~mm}$ larger than sagittal diameter of AA in patients without significant AS. The aortic diameter according to area is similar than aortic diameter in sagittal view and aortic diameter according to perimeter is $0.93 \mathrm{~mm}$ larger than that in sagittal view and $1 \mathrm{~mm}$ smaller than this diameter in coronal view. D1 is the smallest aortic diameter [5]. Mean RCA distance from AA is $1 \mathrm{~mm}$ greater than mean LM distance from AA and mean left coronary cusp is about $1.2 \mathrm{~mm}$ smaller than distance of LM from AA.

The results of our study showed that D1 is significantly smaller than D3, so the annulus in short-axis (smaller diameter) is smaller than the AA in sagittal view and they are not the same measurement and can not be replaced by each other. Also, the difference between D2 and D4 is not statistically significant, so the AA in coronal view and short-axis view (greater diameter) are the same.

The AA in sagittal view is about $2 \mathrm{~mm}$ smaller than the AA in coronal view. So the AA in non-stenotic valves is not round and is oval shape.

Although the difference between D3 and D5 is not statistically significant, but mean difference is $0.66 \mathrm{~mm}$ and $\mathrm{P}=0.07$, it is probable that with a larger study population and especially in patients with significant AS with left ventricular hypertrophy, this difference would be significant. With respect to this fact that for self-expandable valves, ventricular end is larger than mid-portion diameter[2], this measurement is very important. It is notable that height of skirt is $12 \mathrm{~mm}$ in Cor valve prosthesis and between 7.74 and $8.67 \mathrm{~mm}$ for Edward Sapien valves[3]. The Edwards SAPIEN valve optimal position is with the ventricular side of the prosthesis located2 $-4 \mathrm{~mm}$ below the annulus, while the CoreValve is recommended to have the ventricular edge of the prosthesis placed 5- $10 \mathrm{~mm}$ below the aortic valve annular plane.

D perimeter in our study is larger than $\mathrm{D}$ area which is comparable with study by Blank $\mathrm{P}$ et $\mathrm{Al}[6]$.

In our study, AA was $23.06 \pm 2.87 \mathrm{~mm}$ in sagittal view and $25.03 \pm 3.69 \mathrm{~mm}$ in coronal view by TEE 3D echocardiogeaphy.

Our result is comparable with the results of the study by Omar Khalique et al which showed that max aortic annular diameter by TEE 3D echo was $25.3 \pm 2.6 \mathrm{~mm}$ and minimal aortic diameter was $21.9 \pm$ $2.2 \mathrm{~mm}, \mathrm{D}$ according to area was $23.4 \pm 2.2 \mathrm{~mm}$ and $\mathrm{D}$ according to perimeter was $23.8 \pm 2.2 \mathrm{~mm}, \mathrm{D}$ perimeter was $24.1 \pm 2.2 \mathrm{~mm}$ by CT scan[1]. 
Transcatheter aortic valve implantation bioprostheses are typically oversized by $5-30 \%$ relative to the aortic valve annulus diameter[7].

The CoreValve recommendations suggest an oversizing percentage between 7 and $30 \%$ and Edwards SAPIEN between 4 and 27\%[7].

Most procedures are performed according to the annular diameter measurement by 2D TTE or TOE, but these methods assume annular circularity, which may result in erroneous dimensions inpatients whose annuli are more oval shaped[7].

Cor valve self-expandable prosthesis have two size of 26 and $29 \mathrm{~mm}$ according to external diameter of the ventricular end with midportion diameters of 22 and $24 \mathrm{~mm}$ which are used for annulus $20-23 \mathrm{~mm}$ and 24-27 mm respectively[2]. Edward-Sapien balloon expandable prosthesis $23 \mathrm{~mm}$ are used for AA 18-21 and $26 \mathrm{~mm}$ for AA 22-25 mm for prevention of paravalvular leakage[2].Due to this fact that coronal diameter is about $2 \mathrm{~mm}$ greater than sagittal diameter, this oversizing can be explained.

Indeed, the reason for oversize may be explained by this fact that the traditional measurements of AA is made in sagittal view and this is the smallest aortic diameter. In clinical practice, balloon expandable devices are largely sized on the basis of annular area, with self-expandable devices relying on perimeter [5].

D perimeter is between $D$ coronal and $D$ sagittal, it is about $1 \mathrm{~mm}$ smaller than $D$ coronal and 0.93 larger than $\mathrm{D}$ sagittal.

In our study, mean distance between LM ostium and AA is $14.2 \pm 2.94 \mathrm{~mm}$ and mean distance between right coronary ostium and $A A=15.25 \pm 3.60 \mathrm{~mm}$. As the best of our knowledge,our study is the first study focusing on distance of LM and RCA from annulus by 3D echocardiography.

In a study by Cavalcanti et al the mean distance measured from the orifice of the left coronary artery to the basal attachment of the corresponding leaflet was $12.6 \pm 2.61 \mathrm{~mm}$, and for the right coronary artery it was $13.2 \pm 2.64 \mathrm{~mm}$ in normal heart post mortem study [9] .

In a recent study examining the aortic root with multislice computed tomography in 169 patients (150 patients with no or mild AS and 19 patients with moderate or severe AS), the mean distance from the level of the basal attachment point of the aortic valvar leaflets to the ostium of the left coronary and right coronary artery was $14.42 .9 \mathrm{~mm}$ and $17.23 .3 \mathrm{~mm}$, respectively [8]. There were no significant differences in the mean distances between patients with or without severe AS.

The possible reason for the difference between our study and Cavalcanti study is that they studied postmortem patients.

The minimum distance between the coronary ostia and the aortic valve annular plane should be $\geq 10-$ $11 \mathrm{~mm}$ for both commercially available valves [2]. 
Length of left coronary cusp is about $1 \mathrm{~mm}$ shorter than distance of LM from AA, and distance of RCA is about $1 \mathrm{~mm}$ longer than distance of LM from AA.

\section{Conclusion:}

$A A$ is is not completely round in non-stenotic aortic valves and is larger in coronal than sagittal view, AA according to area is not different with annulus in sagittal view and AA according to perimeter is between annulus in sagittal and coronal views. Under sizing of AA for TAVI may cause complications like paravalvular leakage [10].

\section{Abbreviations}

\section{TEE}

Transesophageal Echocardiography

AS

Aortic Stenosis

AA

Aortic Annulus

TAVI

Transaortic Valve Implantation

TTE

Transthoracic Echocardiography

TOE

transoesophageal echocardiography

MSCT

multi-slice computed tomography

LVEF

Left Ventricular Ejection Fraction

LM

left main

RCA

right coronary artery

LVOT

left ventricular outflow tract

RBG

Red, Blue, Green

LA

Left atrium

AO

Aorta 
LVDD

Left Ventricular Dimension Diastolic

LVDS

Left Ventricular Dimension Systolic

IVS

interventricular septum

PW

posterior wall

RVD

Right Ventricular Dimension

TAPSE

Tricuspid Annular Plane Systolic Excursion

GRAO

Gradient Aorta

\section{Declarations}

Ethics approval and consent to participate

This study was approved by Tehran Heart Center.

Consent for publication

Not applicable

Availability of data and materials

Not applicable

Competing interests

The authors declare that they have no competing interests

Funding

None

Authors' contributions

Study concept and design: H.S.; analysis and interpretation of data: A.J.O.; drafting of the manuscript: M.A.; critical revision of the manuscript fot important intellectual content: M.A., H.S., and A.J.; statistical analysis: A.J.O., A.J..

Acknowledgements 
Special thanks to Tehran Heart Center employees for supporting the authors in collecting data and the patients that accepted to participate in our study.

\section{Tables}

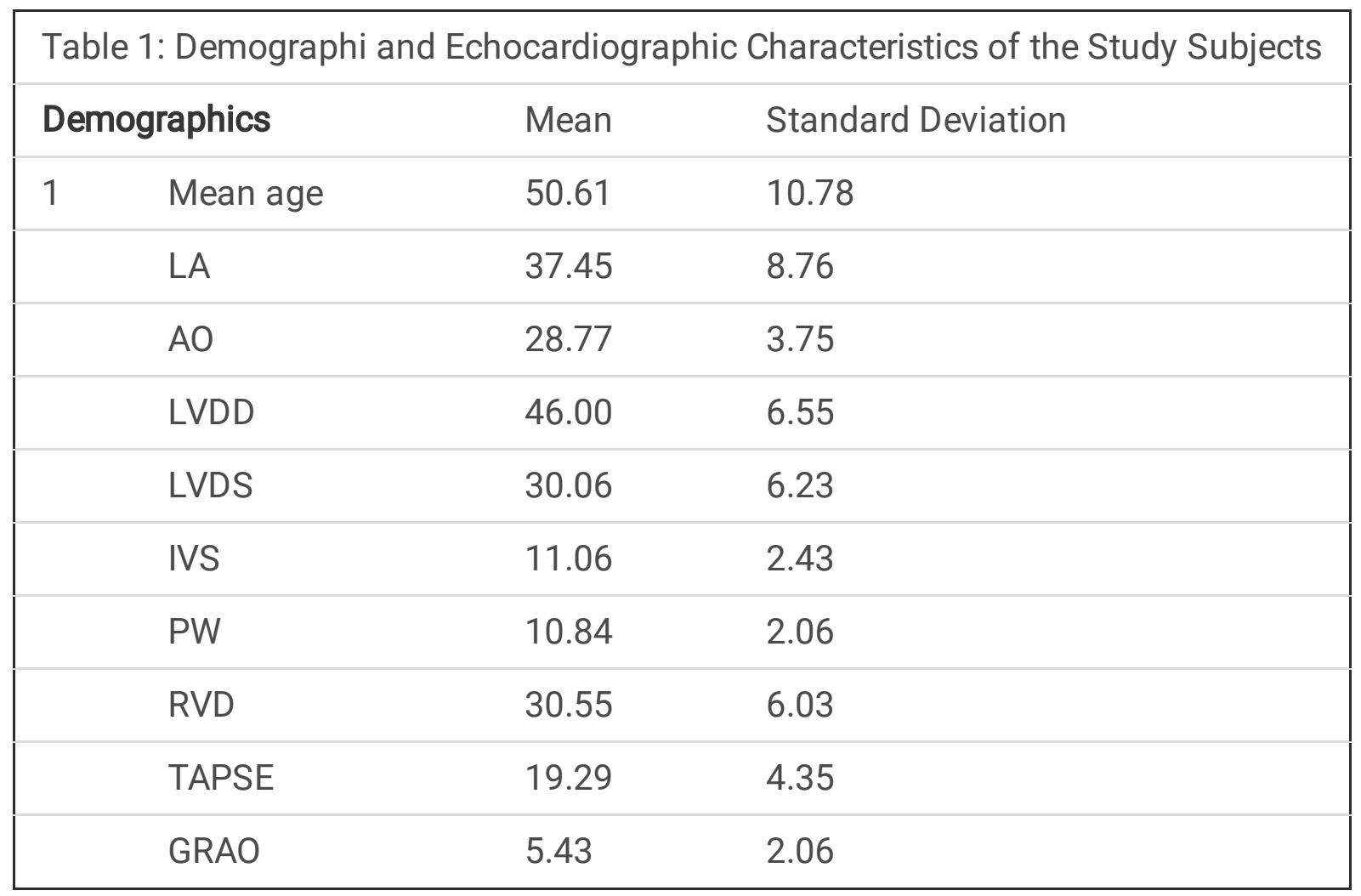

LA: Left atrium ; AO: Aorta ; LVDD: Left Ventricular Dimension Diastolic ; LVDS: Left Ventricular Dimension Systolic ; IVS: interventricular septum thickness ; PW: posterior wall thickness ; RVD: Right Ventricular Dimension ; TAPSE: Tricuspid Annular Plane Systolic Excursion ; GRAO: Gradient Aorta 


\begin{tabular}{|lll|}
\hline Table 2: & Echocardiographic Measurements of Aortic Valve of the Study Subjects \\
\hline & Mean & Standard Deviation \\
\hline LM distance & 14.20 & 2.94 \\
\hline RCA distance & 15.26 & 3.61 \\
\hline Area of AA & 4.31 & .95 \\
\hline Perimeter of AA & 7.54 & .85 \\
\hline Left coronary cusp length & 12.75 & 2.12 \\
\hline D1 & 22.44 & 2.62 \\
\hline D2 & 24.92 & 3.15 \\
\hline D3 & 23.06 & 2.87 \\
\hline D4 & 25.03 & 3.69 \\
\hline D5 & 22.39 & 3.12 \\
\hline D6 & 24.25 & 5.70 \\
\hline
\end{tabular}

LM: Left Main, RCA: Right Coronary Artery, AA: Aotrtic Annulus

\section{References}

1. Khalique, O.K., et al., Aortic annular sizing using a novel 3-dimensional echocardiographic method: use and comparison with cardiac computed tomography. Circ Cardiovasc Imaging, 2014. 7(1): p. $155-63$.

2. Zamorano, J.L., et al., EAE/ASE recommendations for the use of echocardiography in new transcatheter interventions for valvular heart disease. J Am Soc Echocardiogr, 2011. 24(9): p. 937-65.

3. Piazza, N., et al., Anatomy of the aortic valvar complex and its implications for transcatheter implantation of the aortic valve. Circ Cardiovasc Interv, 2008. 1(1): p. 74-81.

4. Sadeghian, H., et al., Ventricular asynchrony of time-to-peak systolic velocity in structurally normal heart by tissue Doppler imaging. Echocardiography, 2010. 27(7): p. 823-30.

5. Blanke, P., et al., Computed Tomography Imaging in the Context of Transcatheter Aortic Valve Implantation (TAVI)/Transcatheter Aortic Valve Replacement (TAVR): An Expert Consensus Document of the Society of Cardiovascular Computed Tomography. JACC Cardiovasc Imaging, 2019. 12(1): p. 1-24.

6. Blanke, P., et al., Oversizing in transcatheter aortic valve replacement, a commonly used term but a poorly understood one: dependency on definition and geometrical measurements. J Cardiovasc Comput Tomogr, 2014. 8(1): p. 67-76. 
7. Zamorano, J.L., A. Goncalves, and R. Lang, Imaging to select and guide transcatheter aortic valve implantation. Eur Heart J, 2014. 35(24): p. 1578-87.

8. Tops, L.F., et al., Noninvasive evaluation of the aortic root with multislice computed tomography implications for transcatheter aortic valve replacement. JACC Cardiovasc Imaging, 2008. 1(3): $p$. 321-30.

9. Cavalcanti JS, de Melo MN, de Vasconcelos RS. Morphometric and topographic study of coronary ostia. Arq Bras Cardio. 2003;81:359 -362,355-358.

10. Hakimeh Sadeghian, Mohammad Moein Ashrafi. Transcatheter Aortic Valve Implantation - Tavi. Curr Trends Clin Med Imaging.0060 2019; 3(3): 555614.DOI: 10.19080/CTCMI.2019.03.555614

\section{Figures}




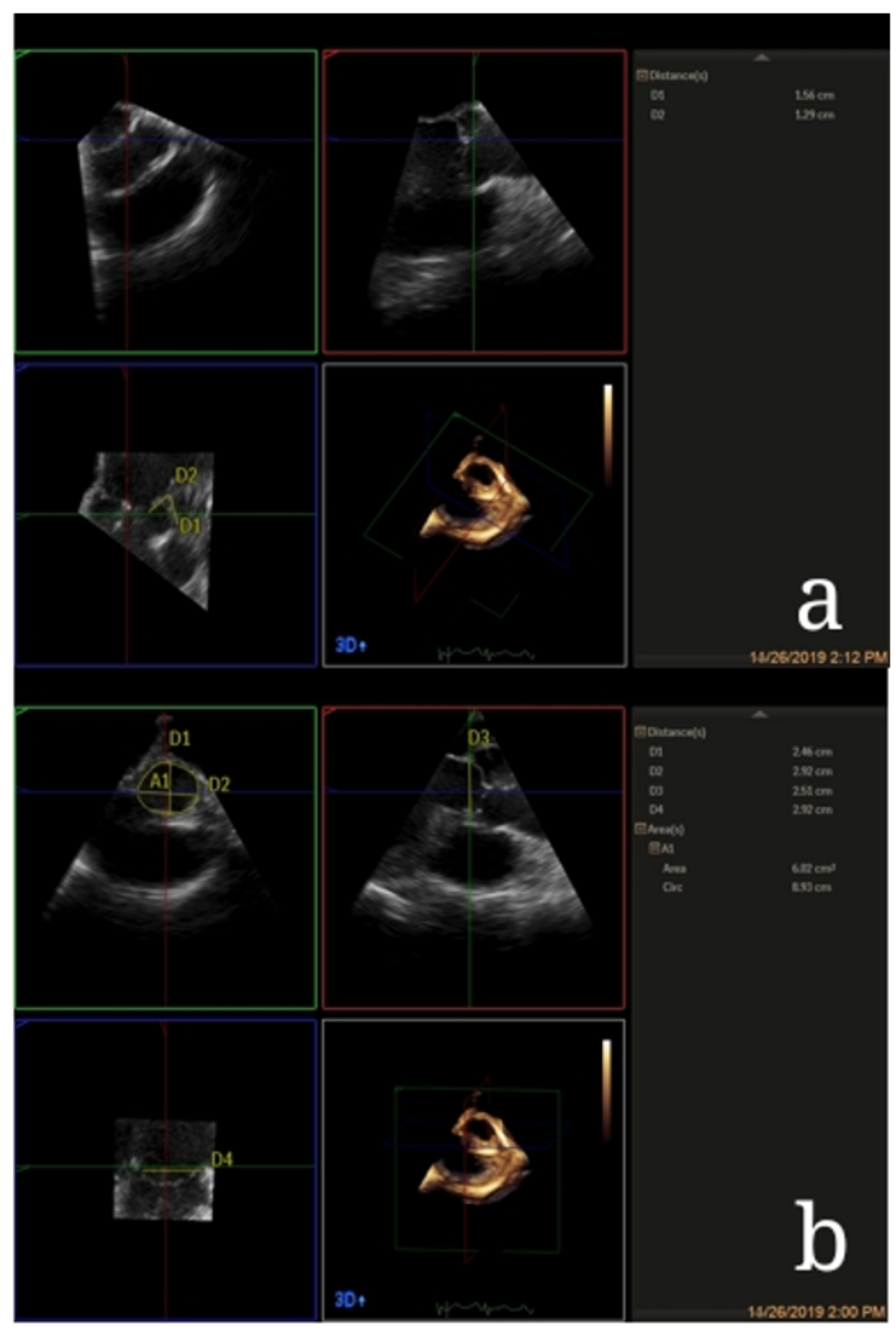

\section{Figure 1}

Distance of orifice of left main from aortic annulus and left coronary cusp length in coronal plane (a), Full-volume of aortic valve by 3D echocardiography in short-axis view in single beat for measurement of aortic annulus, area and perimeter (b). 


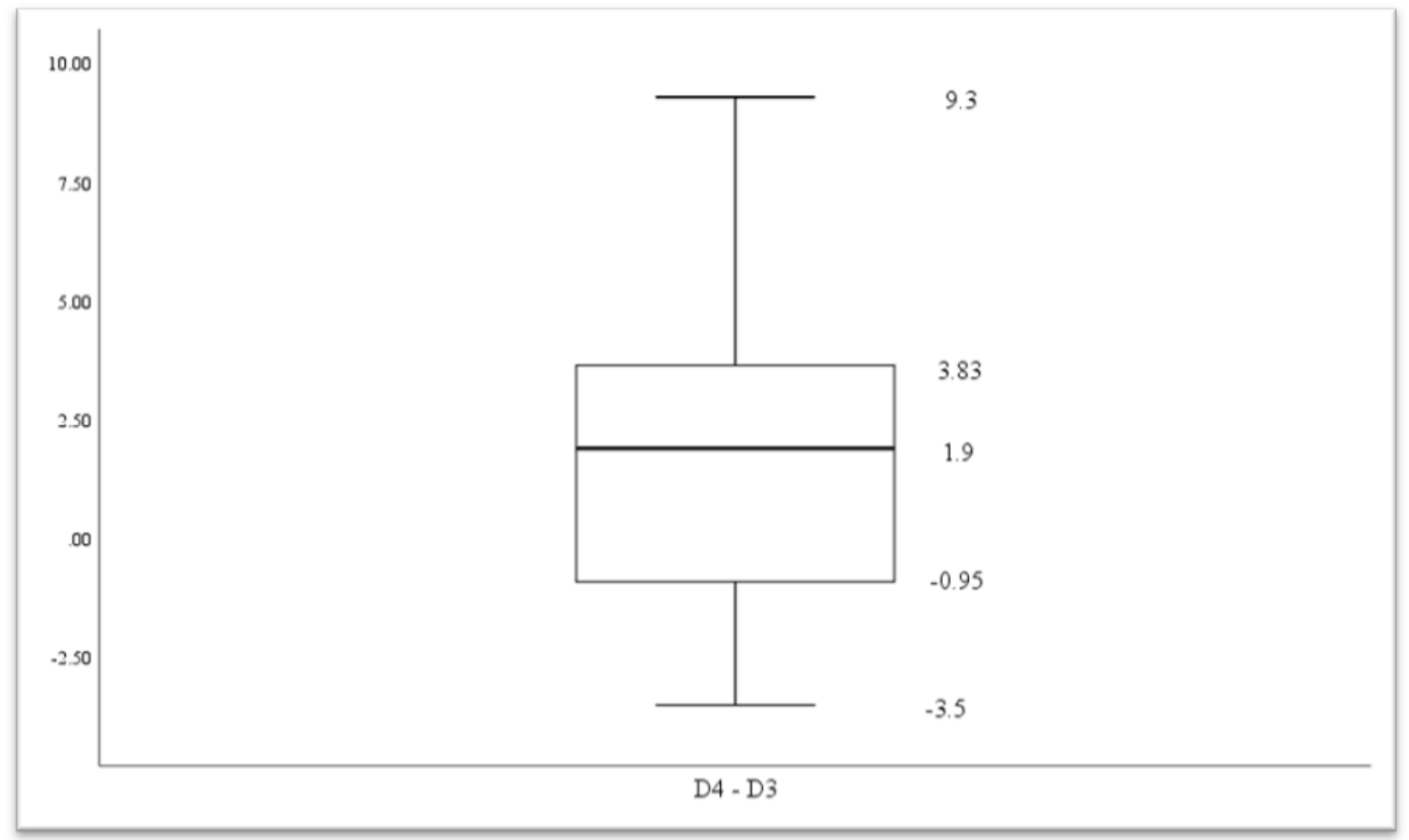

Figure 2

Empiric distribution of difference D4 and D3 in each subject by a plot, median difference is $1.9 \mathrm{~mm}$, maximum difference is $9.3 \mathrm{~mm}$ and minimum difference is $-3.5 \mathrm{~mm}$. 